\title{
Inclusive and differential cross-sections measurements in the single top tW e-mu channel with CMS
}

\author{
Alejandro Soto Rodriguez for the CMS collaboration \\ University of Oviedo, Spain \\ E-mail: alejandro.soto.rodriguez@cern.ch
}

Inclusive and normalised differential cross sections measurements are presented for the production of single top quarks in association with a $\mathrm{W}$ boson, in proton-proton collisions at a centre-ofmass energy of $13 \mathrm{TeV}$. Events containing one muon and one electron in the final state are analysed. For the inclusive measurement, a multivariate discriminant, exploiting the kinematic properties of the events, is used to separate the signal from the dominant tt background. For the differential measurements, a fiducial region is defined according to the detector acceptance, and the requirement of exactly one b-tagged jet. The resulting distributions are unfolded to particle-level and compared with predictions calculated at next-to-leading order in perturbative QCD. Within current uncertainties, all predictions agree with the data.

\footnotetext{
*** The European Physical Society Conference on High Energy Physics (EPS-HEP2021), *** *** 26-30 July $2021 * * *$

*** Online conference, jointly organized by Universität Hamburg and the research center DESY ***
} 


\section{Introduction}

Single top quarks were observed for the first time by the D0 [1] and CDF [2] Collaborations at the Fermilab Tevatron collider. There are three main production modes in proton-proton (pp) collisions: the exchange of a virtual $\mathrm{W}$ boson ( $t$ channel), the production and decay of a virtual $\mathrm{W}$ boson ( $s$ channel), and the associated production of a top quark and a $\mathrm{W}$ boson (tW channel).

The study of the tW process provides a unique opportunity to probe the standard model (SM) and its extension through its interference with top quark pair production (tî) at NLO in QCD. In this analysis, two schemes are defined to avoid double counting issues: diagram removal (DR), in which all doubly resonant diagrams are removed from the matrix element (ME) calculation, and diagram subtraction (DS), in which a gauge invariant term is introduced in the ME calculation that locally cancels the doubly resonant diagrams. The $\mathrm{tW}$ production cross section is computed at approximate next-to-next-to-leading order (NNLO). The corresponding theoretical prediction for the tW cross section in pp collisions at $\sqrt{s}=13 \mathrm{TeV}$, assuming a top quark mass $\left(m_{\mathrm{t}}\right)$ of $172.5 \mathrm{GeV}$ [3], is

$$
\sigma_{\mathrm{tW}}^{\mathrm{SM}}=71.7 \pm 1.8(\text { scale }) \pm 3.4(\mathrm{PDF}) \mathrm{pb} .
$$

This document reports the first measurement from the CMS Collaboration of the inclusive [4] and differential [5] cross sections of the tW process in pp collisions at $\sqrt{s}=13 \mathrm{TeV}$. The measurement is performed using final states with one electron and one muon of opposite charge. The analysed data was recorded by the CMS detector [6] during 2016 and corresponds to $35.9 \mathrm{fb}^{-1}$ of integrated luminosity.

\section{Event selection}

The analysis uses the $\mathrm{e}^{ \pm} \mu^{\mp}$ channel of the tW process, which corresponds to a final state with one electron and one muon of opposite charge, the two corresponding neutrinos and a jet resulting from the fragmentation of the bottom quark. The main background contribution arises from the $\bar{t} \bar{t}$ process, which can give very similar final states with only one additional b quark. To select signal events and reduce the background contributions an event selection is performed.

Events are required to pass either a dilepton or single-lepton trigger. The efficiency for the combination of the single-lepton and dilepton triggers is found to be $\simeq 98 \%$. Leptons (electrons or muons) are required to be well isolated and to have $p_{\mathrm{T}}>20 \mathrm{GeV}$ and $|\eta|<2.4$. In events with more than two leptons passing the selection, the two with the largest $p_{\mathrm{T}}$ are selected for further study. Jets are reconstructed using the anti- $\kappa_{\mathrm{T}}$ clustering algorithm with a distance parameter of 0.4 . Jets are required to have $p_{\mathrm{T}}>30 \mathrm{GeV}$, and $|\eta|<2.4$. To avoid double counting issues, jets within a cone of $\Delta R=0.4$ with respect to the selected leptons are not considered. An additional category of jets called "loose jets" is defined to be jets passing the above selection but with $p_{\mathrm{T}}$ between 20 and $30 \mathrm{GeV}$.

Some sources of background, such as Drell-Yan (DY), do not contain jets originated from a $b$ quark. The identification of jets originating from $b$ quarks results in a significant reduction in background. Jets are identified as b jets using the algorithm CSVv2 [7], with a working point that yields identification efficiencies of $\simeq 70 \%$ and misidentification probabilities of about $1 \%$ and $15 \%$ [7] for light-flavor jets and $\mathrm{c}$ jets respectively. 
Events are classified as belonging to the $\mathrm{e}^{ \pm} \mu^{\mp}$ final state if the two leptons with larger $p_{\mathrm{T}}$ (leading leptons) passing the above selection criteria are an electron and a muon of opposite charge. We also require that the leading lepton has $p_{\mathrm{T}}>25 \mathrm{GeV}$. To reduce the contamination from low mass resonances, the invariant mass of the dilepton pair must be greater than $20 \mathrm{GeV}$. Different regions for the inclusive and differential measurements are defined based on the number of jets and b-tagged jets. Figure 1 shows this distribution and the data/Monte Carlo (MC) comparison. For the inclusive measurement the regions with one $b$-tagged jet ( $1 \mathrm{j} 1 \mathrm{~b})$, two jets and one of them b-tagged $(2 \mathrm{j} 1 \mathrm{~b})$ and two $b$-tagged jets $(2 \mathrm{j} 2 \mathrm{~b})$ are used, while for the differential measurements only the $1 \mathrm{j} 1 \mathrm{~b}$ region is used.

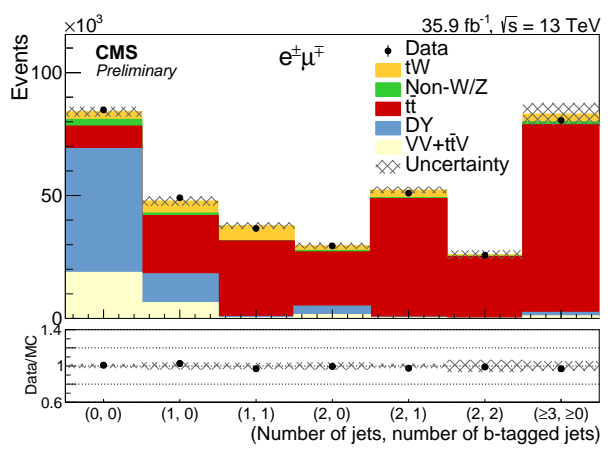

Figure 1: Yields observed in data, compared with those expected from simulation, as a function of the number of jets and number of b-tagged jets for events passing the baseline dilepton selection. The error band includes the statistical and all systematic uncertainties, except those from background normalisation. The bottom of each panel shows the ratios of data to the sum of the expected yields [4].

\section{Inclusive measurement}

Following the event selection the data sample in the $1 \mathrm{j} 1 \mathrm{~b}$ region consists primarily of $t \bar{t}$ events with a significant number of tW events. Given that there is no single observable that discriminates between $\mathrm{t} \overline{\mathrm{t}}$ and $\mathrm{tW}$ events, two boosted decision trees (BDT) are trained, one in the $1 \mathrm{j} 1 \mathrm{~b}$ region and the other in the $2 \mathrm{j} 1 \mathrm{~b}$ region, using kinematic properties of the events, to discriminate between signal and the dominant $t \bar{t}$ background. The BDT implementation is provided by the TMVA [8] package. The $2 \mathrm{j} 2 \mathrm{~b}$ region is used as a $\overline{\mathrm{t}} \overline{\mathrm{t}}$ control region to constrain this main source of background.

The signal is extracted by performing a simultaneous maximum likelihood (ML) fit to the distributions of the BDT output in the $1 \mathrm{j} 1 \mathrm{~b}$ and $2 \mathrm{j} 1 \mathrm{~b}$ regions and the subleading jet $p_{\mathrm{T}}$ in the $2 \mathrm{j} 2 \mathrm{~b}$ region. The binning of the BDT outputs is chosen such that each bin contains approximately the same amount of $t \bar{t}$ events. This ensures that enough background events populate all the bins, helping to constrain the systematic uncertainties. Figure 2 shows the postfit distributions of the three variables that enter in the fit.

The measured inclusive cross section of the tW process that results in the best fit to the data is

$$
\sigma_{\mathrm{tW}}^{\mathrm{exp}}=63.1 \pm 1.8(\text { stat }) \pm 6.4 \text { (syst) } \pm 2.1 \text { (lumi) pb, }
$$

consistent with the SM expectations of Eq. 1. 

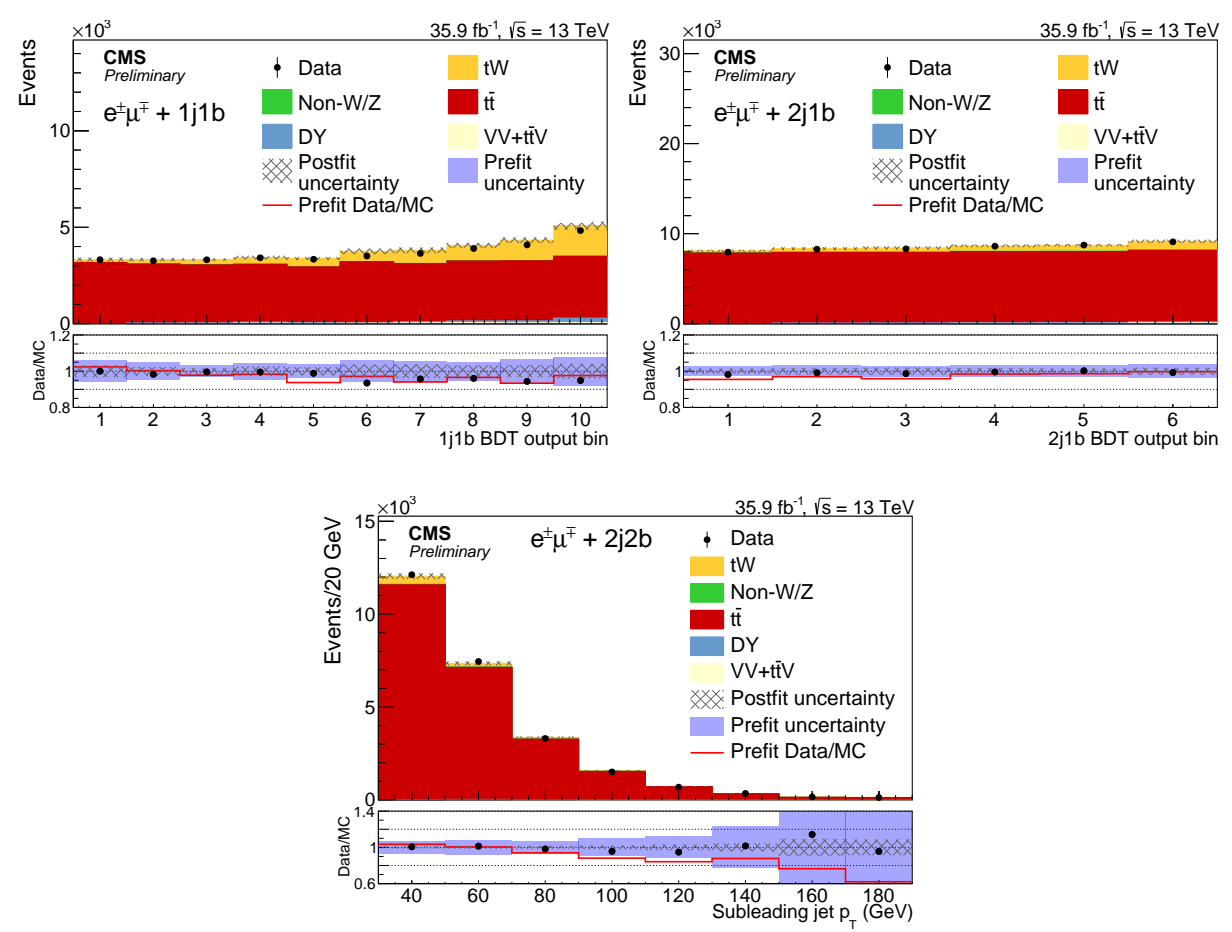

Figure 2: Comparison of the BDT output in the $1 \mathrm{j} 1 \mathrm{~b}$ (upper left) and $2 \mathrm{j} 1 \mathrm{~b}$ (upper right) regions and the $p_{\mathrm{T}}$ of the subleading jet in the $2 \mathrm{j} 2 \mathrm{~b}$ region (lower) distributions after the fit is performed for the observed data and simulated events. The error band includes the statistical and systematic uncertainties. The bottom of each panel shows the ratios of data to the prediction from simulations (line) and from the fit (dots), together with their corresponding uncertainties (solid and hatched band, respectively) [4].

\section{Differential measurement}

For the differential measurement only the $1 \mathrm{j} 1 \mathrm{~b}$ region is used with an additional criterion on the number of loose jets (Fig. 3). Selecting events with zero identified loose jets enhances the signal to background ratio. Therefore, to minimise the relative contribution from the $\mathrm{t} \overline{\mathrm{t}}$ background, the signal region is defined as that with events in the $1 \mathrm{j} 1 \mathrm{~b}$ region and without loose jets.

The signal is extracted by subtracting background to data. Unfolding techniques are used to take into account migration effects when extrapolating to the fiducial region at particle level. The fiducial region is defined by the same selection requirements employed in the event selection but applied on particle-level objects. The differential cross sections are measured as function of the leading lepton $p_{\mathrm{T}}$, jet $p_{\mathrm{T}}, \Delta \phi\left(\mathrm{e}^{ \pm}, \mu^{\mp}\right), p_{\mathrm{Z}}\left(\mathrm{e}^{ \pm}, \mu^{\mp}, j\right), m\left(\mathrm{e}^{ \pm}, \mu^{\mp}, j\right)$ and $m_{\mathrm{T}}\left(\mathrm{e}^{ \pm}, \mu^{\mp}, j, p_{\mathrm{T}}^{\text {miss }}\right)$ (the definition of these variables can be found in [5]) and normalised to the fiducial cross section.

Figure 4 shows the normalised differential cross sections as a function of the leading lepton $p_{\mathrm{T}}$ and $\Delta \phi\left(\mathrm{e}^{ \pm}, \mu^{\mp}\right)$. Good agreement, within the uncertainties, with the predictions from POWHEG DR, POWHEG DS and MADGRAPH5_aMC@NLO DR is observed. 


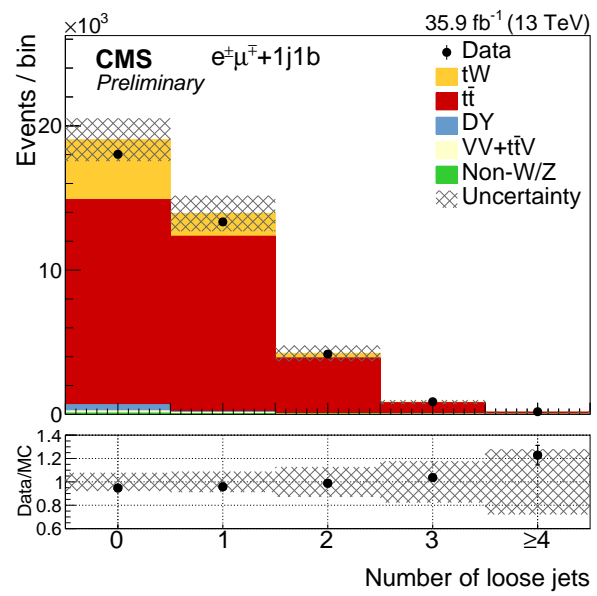

Figure 3: Yields observed in data, compared with those expected from simulation, as a function of the number of loose jets for events passing the $\mathrm{e}^{ \pm} \mu^{\mp}$ selection in the $1 \mathrm{j} 1 \mathrm{~b}$ region. The bottom of the panel shows the ratio of data to the sum of the expected yields [5].
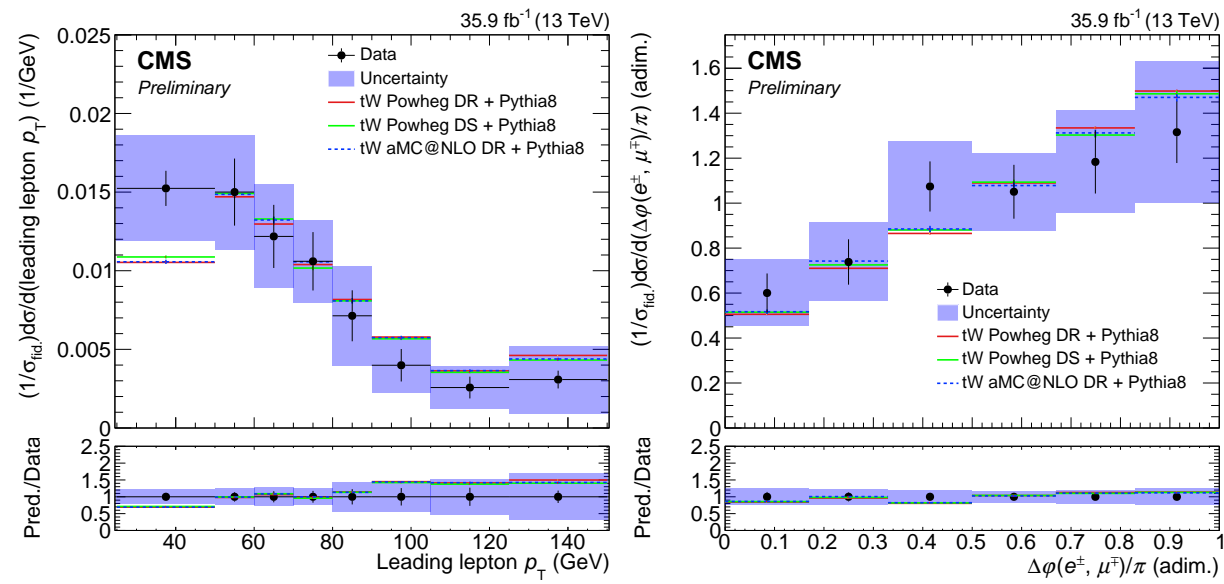

Figure 4: Normalised differential tW production cross section as a function of the $p_{\mathrm{T}}$ of the leading lepton and $\Delta \phi\left(\mathrm{e}^{ \pm}, \mu^{\mp}\right)$ (right). The solid band represents the total uncertainty. Predictions from POWHEG and MADGRAPH5_aMC@NLO are also shown. In the bottom panel, the ratio between predictions and data is shown [5].

\section{Summary}

A measurement of the inclusive and differential cross sections of the tW process at $13 \mathrm{TeV}$ with the CMS detector is presented. The analysis employs the $\mathrm{e}^{ \pm} \mu^{\mp}$ channel and classifies the events in terms of the number of jets and b-tagged jets to perform the measurement. The inclusive cross section is obtained using a maximum likelihood fit to the distribution of the boosted decision tree discriminants in two categories, and the subleading jet $p_{\mathrm{T}}$ in a third category. The measured cross section is found to be $63.1 \pm 1.8$ (stat) \pm 6.4 (syst) \pm 2.1 (lumi) pb, achieving a relative uncertainty of $11 \%$ in agreement with the SM. Finally, the measured differential cross sections are, in general, consistent with the expectations from the models used to simulate the tW signal. 


\section{References}

[1] D0 Collaboration, “Observation of single top quark production”, Phys. Rev. Lett. 103 (2009) 092001, doi:10.1103/PhysRevLett.103.092001, arXiv:0903.0850.

[2] CDF Collaboration, "First observation of electroweak single top quark production", Phys. Rev. Lett. 103 (2009) 092002, doi:10.1103/PhysRevLett.103.092002, arXiv:0903.0885.

[3] N. Kidonakis, "Theoretical results for electroweak-boson and single-top production", in Proceedings of 23rd International Workshop on Deep-Inelastic Scattering and Related Subjects (DIS 2015), Dallas U.S.A. (2015), volume DIS2015, pg. 170, arXiv:1506.04072.

[4] CMS Collaboration, "Measurement of the production cross section for single top quarks in association with W bosons in proton-proton collisions at $\sqrt{s}=13 \mathrm{TeV}$ ", JHEP 10 (2018) 117, doi:10.1007/JHEP10(2018)117, arXiv:1805.07399.

[5] CMS Collaboration, "Measurement of differential cross sections for single top quark production in association with a W boson at $\sqrt{s}=13 \mathrm{TeV}$ ", CMS Physics Analysis Summary CMS-PAS-TOP-19-003, CERN, 2020.

[6] CMS Collaboration, "The CMS experiment at the CERN LHC", JINST 3 (2018) S08004, doi:10.1088/1748-0221/3/08/s08004.

[7] CMS Collaboration, "Identification of heavy-flavour jets with the CMS detector in pp collisions at 13 TeV”, 2018 JINST 13 P05011, arXiv:1712.07158.

[8] H. Voss, A. Höcker, J. Stelzer, and F. Tegenfeld, "TMVA, the toolkit for multivariate data analysis with ROOT", in XI International Workshop on Advanced Computing and Analysis Techniques in Physics Research, p. 040. SISSA, 2007, arXiv:physics/0703039, PoS(ACAT2007)040. 